\title{
Noradrenergic Activation of the Basolateral Amygdala Modulates Consolidation of Object Recognition Memory
}

\author{
Benno Roozendaal, Nicholas A. Castello, Gustavo Vedana, Areg Barsegyan, and James L. \\ McGaugh \\ Center for the Neurobiology of Learning and Memory, Department of Neurobiology and Behavior, \\ University of California, Irvine, Irvine, CA 92697-3800
}

\begin{abstract}
Noradrenergic activation of the basolateral complex of the amygdala (BLA) modulates the consolidation of memory for many kinds of highly emotionally arousing training tasks. The present experiments investigated whether posttraining noradrenergic activation of the BLA is sufficient to enable memory consolidation of a low-arousing training experience. Sprague-Dawley rats received intra-BLA infusions of norepinephrine, the $\beta$-adrenoceptor antagonist propranolol or saline immediately after either 3 or 10 min of object recognition training. Saline-infused controls exhibited poor 24-h retention when given 3 min of object recognition training and good retention when given $10 \mathrm{~min}$ of training. Norepinephrine administered after $3 \mathrm{~min}$ of object recognition training produced dose-dependent enhancement of 24-h object recognition memory whereas propranolol administered after $10 \mathrm{~min}$ of training produced dose-dependent impairment of memory. These findings provide evidence that posttraining noradrenergic activation of the BLA enhances memory of a low-arousing training experience that would otherwise not induce long-term memory. Thus, regardless of the degree of emotional arousal induced by an experience, noradrenergic activation of the BLA after the experience ensures that it will be better remembered.
\end{abstract}

\section{Keywords}

Norepinephrine; Propranolol; $\beta$-adrenoceptor; Emotional arousal; Memory modulation

\begin{abstract}
There is extensive evidence that noradrenergic activation of the basolateral complex of the amygdala (BLA) modulates memory consolidation (McGaugh, 2000; 2004; McGaugh \& Roozendaal, 2002). Norepinephrine or the $\beta$-adrenoceptor agonist clenbuterol infused into the BLA immediately posttraining enhance the memory of many emotionally arousing training tasks, including inhibitory avoidance (Ferry, Roozendaal, \& McGaugh, 1999; IntroiniCollison, Miyazaki, \& McGaugh, 1991), contextual fear conditioning (Huff, Wright-Hardesty, Higgins, Matus-Amat, \& Rudy, 2005; LaLumiere, Buen, \& McGaugh, 2003), water-maze spatial training (Hatfield \& McGaugh, 1999) and extinction of contextual fear conditioning (Berlau \& McGaugh, 2005). In contrast, posttraining intra-BLA infusions of $\beta$-adrenoceptor antagonists impair the consolidation of memory for emotionally arousing training experiences (Hatfield \& McGaugh, 1999; Miranda, LaLumiere, Buen, Bermudez-Rattoni, \& McGaugh,
\end{abstract}

Correspondence: James L. McGaugh, 320 QRL - CNLM, UC Irvine, Irvine, CA 92697-3800, Telephone: (949) 824-5401, Fax: (949) 824-2952, EMAIL: JLMCGAUG@UCI.EDU.

Publisher's Disclaimer: This is a PDF file of an unedited manuscript that has been accepted for publication. As a service to our customers we are providing this early version of the manuscript. The manuscript will undergo copyediting, typesetting, and review of the resulting proof before it is published in its final citable form. Please note that during the production process errors may be discovered which could affect the content, and all legal disclaimers that apply to the journal pertain. 
2003). Such findings strongly suggest that noradrenergic activation of the BLA is critically involved in regulating the consolidation of memory of many different kinds of information (McGaugh, 2002; 2004).

Prior studies of the role of noradrenergic activation of the BLA have used highly arousing training conditions, such as footshock, that are known to induce the release of norepinephrine within the amygdala (Hatfield, Spanis, \& McGaugh, 1999; McIntyre, Hatfield, \& McGaugh, 2002; Quirarte, Galvez, Roozendaal, \& McGaugh, 1998). However, it is not known whether posttraining noradrenergic activation of the BLA is sufficient to modulate the consolidation of experiences that do otherwise not induce long-term memory. To address this issue, the present experiments examined the effects of posttraining intra-BLA infusions of norepinephrine or the $\beta$-adrenoceptor antagonist propranolol on rats' 24 -h memory of object recognition training, a task that relies on the spontaneous tendency of rats to explore a novel object more than a familiar one and induces only low levels of emotional arousal (Roozendaal, Okuda, Van der Zee, \& McGaugh, 2006).

Male Sprague-Dawley rats (280-320 $\mathrm{g}$ at time of surgery) from Charles River Laboratories (Wilmington, MA) were housed individually and maintained on a 12-h/12-h light/dark cycle with ad libitum access to food and water. After at least 1 week of adaptation they received surgical implantation of cannulae aimed at the BLA. They were anesthetized with nembutal $(50 \mathrm{mg} / \mathrm{kg}$ of body weight, i.p.), given atropine sulfate $(0.4 \mathrm{mg} / \mathrm{kg}$, i.p.) to maintain respiration, and were subsequently injected with $3.0 \mathrm{ml}$ of saline. The skull was positioned in a stereotaxic frame (Kopf Instruments, Tujunga, CA), and two stainless-steel guide cannulae (15 mm; 23 gauge; Small Parts, Inc, Miami Lakes, FL) were implanted bilaterally with the cannula tips 2.0 $\mathrm{mm}$ above the BLA. The coordinates were based on the atlas of Paxinos and Watson (2005): anteroposterior, $-2.8 \mathrm{~mm}$ from bregma; mediolateral, $\pm 5.0 \mathrm{~mm}$ from midline; dorsoventral, $-6.5 \mathrm{~mm}$ from skull surface; incisor bar $-3.3 \mathrm{~mm}$ from interaural. Stylets inserted into each cannula to maintain patency were removed only for the drug infusions. After surgery, the rats were retained in an incubator to recover from anesthesia and then allowed 10 days of recovery prior to training. All procedures were in compliance with NIH guidelines and were approved by the Institutional Animal Care and Use Committee of the University of California, Irvine.

The training apparatus was a grey wooden box $(40 \mathrm{w} \times 40 \mathrm{~d} \times 40 \mathrm{~h} \mathrm{~cm})$ placed in a dimly illuminated room. The floor of the box was covered with sawdust. The objects to be discriminated were white glass light bulbs $(6 \mathrm{~cm}$ diameter, $11 \mathrm{~cm}$ length) and transparent glass vials $(5.5 \mathrm{~cm}$ diameter, $5 \mathrm{~cm}$ height). All rats were handled briefly, each day for 7 days prior to training. On the training trial, the rat was placed in the box and allowed to explore two identical objects (A1 and A2) for either $3 \mathrm{~min}$, for the norepinephrine experiment, or $10 \mathrm{~min}$, for the propranolol experiment. Between the training of each rat, the sawdust was stirred and the objects were thoroughly cleaned with $70 \%$ ethanol. On the 24-h retention test, one copy of the familiar object (A3) and a new object (B) were placed in the same location as stimuli during the training trial. The rat was placed in the apparatus for $3 \mathrm{~min}$ and the time spent exploring each object and the total time spent exploring both objects were recorded. Exploration of an object was defined as pointing the nose to the object at a distance of $<1 \mathrm{~cm}$ and/or touching it with the nose (Okuda, Roozendaal, \& McGaugh, 2004). The discrimination index used to assess memory was calculated as the difference in time exploring the novel and familiar object, expressed as the ratio of the total time spent exploring both objects. In previous studies we determined that with 3 min of object recognition training significant memory is displayed $1 \mathrm{~h}$ after training but not after $24 \mathrm{~h}$. In contrast, with 10 min of training significant retention is found at $24 \mathrm{~h}$ (Bermudez-Rattoni, Okuda, Roozendaal, \& McGaugh 2005; Okuda et al., 2004; Roozendaal et al., 2006). Thus, 3 min of training was used for the norepinephrine-treated groups in order to assess possible memory enhancement and $10 \mathrm{~min}$ of training was used for the propranolol-treated groups in order to assess memory impairment. 
Norepinephrine $(0.3,1.0$ or $3.0 \mu \mathrm{g}$ in $0.2 \mu \mathrm{l}$; Sigma-Aldrich, St. Louis, MO) or the $\beta$ adrenoceptor antagonist propranolol $(0.1,0.3$ or $1.0 \mu \mathrm{g}$ in $0.2 \mu \mathrm{l}$; Sigma-Aldrich) were dissolved in saline and infused into the BLA immediately after object recognition training. The animals were gently restrained and bilateral infusions of the drug or an equivalent volume of saline were administered into the BLA via 30-gauge injection needles connected to $10-\mu 1$ Hamilton microsyringes by polyethylene (PE-20) tubing. The injection needles protruded 2.0 $\mathrm{mm}$ beyond the cannula tips and a $0.2-\mu \mathrm{l}$ injection volume per hemisphere was infused over a period of $25 \mathrm{~s}$ by an automated syringe pump (Sage Instruments, Boston, MA). The injection needles were retained within the cannulae for an additional $20 \mathrm{~s}$ to maximize diffusion and to prevent backflow of drug into the cannulae. The infusion volume was based on previous findings from our laboratory indicating that the adjacent central amygdala is not affected (Roozendaal \& McGaugh, 1996, 1997).

For histology, the rats were perfused with $4 \%$ formaldehyde and their brains were sectioned at 50- $\mu \mathrm{m}$ thickness, stained with cresyl violet, and examined by light microscopy. Data of rats with injection needle placements outside the BLA or with extensive tissue damage at the injection needle site were excluded from analyses. A representative photomicrograph of a needle track terminating within the BLA is shown in Figure 1.

On the 3-min training trial the groups that later received posttraining infusions of norepinephrine or saline administered into the BLA did not differ in total object exploration time (one-way ANOVA: $F_{3,29}=1.30 ; P=0.30$ ). Further, one-sample $t$ tests, used to examine whether the discrimination index was different from zero (chance level), indicated that all groups exhibited comparable time exploring each of the two identical objects on the training trial $(P \geq 0.18)$. As is shown in Figure 2A, the saline controls did not express significant 24-h memory of the familiar object (one-sample $t$ test: $\mathrm{t}_{12}=0.29, P=0.78$ ). However, posttraining infusions of norepinephrine into the BLA dose-dependently enhanced retention performance (one-way ANOVA: $F_{3,29}=4.02 ; P<0.05$ ). Post-hoc analysis revealed that the discrimination scores of the groups given 0.3 and $1.0 \mu \mathrm{g}$ doses of norepinephrine, but not the higher dose ( 3 $\mu \mathrm{g})$, were significantly higher than those of saline-treated rats $(0.3 \mu \mathrm{g}: P<0.05 ; 1.0 \mu \mathrm{g}: P<$ 0.01 ), indicating a stronger preference for the novel object. Also, one-sample $t$ tests indicated that rats treated with the 0.3 and $1.0 \mu \mathrm{g}$ doses of norepinephrine exhibited significant exploration preferences for the novel object $\left(\mathrm{t}_{6}=3.44 ; P<0.05, \mathrm{t}_{7}=5.30 ; P<0.005\right.$, respectively). The saline and norepinephrine groups did not differ in total exploration time of the two objects during the retention test $\left(F_{3,29}=1.10 ; P=0.36\right)$.

The groups that later received posttraining intra-BLA infusions of propranolol or saline did not differ in total object exploration time during the 10-min training trial $\left(F_{3,29}=1.50 ; P=\right.$ $0.24)$. Additionally, one-sample $t$ tests indicated that the groups exhibited comparable times exploring each of the two identical objects on the training trial $(P \geq 0.14)$. Figure 2B shows the results of the propranolol and saline-treated rats on the $24 \mathrm{~h}$ retention test. Unlike after 3 min of training, the saline-treated rats displayed a significant preference for the novel object $\left(\mathrm{t}_{8}=6.55, P<0.0005\right)$, whereas posttraining infusions of propranolol into the BLA produced dose-dependent impairment of retention performance $\left(F_{3,29}=8.54 ; P<0.0005\right)$. Post-hoc analysis revealed that all 3 doses of propranolol decreased the discrimination index as compared to that of saline-treated rats (all 3 doses: $P<0.01$ ). One-sample $t$ tests indicated that rats treated with the lower dose of propranolol $(0.1 \mu \mathrm{g})$ still showed a significant exploration preference for the novel object $\left(\mathrm{t}_{8}=2.75 ; P<0.05\right)$ but that rats given the two higher doses of propranolol exhibited no significant exploration preference $\left(\mathrm{t}_{6}=-0.12 ; P=0.91 ; \mathrm{t}_{7}=0.27\right.$; $P=0.80$, respectively). The saline and propranolol groups did not differ in total exploration time of the two objects during the retention trial $\left(F_{3,29}=1.48 ; P=0.24\right)$. 
The main finding of the present experiments is that, in rats given $3 \mathrm{~min}$ of object recognition training, norepinephrine infused into the BLA immediately posttraining induced dosedependent enhancement of 24-h memory of the objects. With these training conditions the saline control animals did not show long-term memory. In a previous study using $3 \mathrm{~min}$ of training we found significant memory at $1 \mathrm{~h}$ following training but not $24 \mathrm{~h}$ later. As discussed above, prior studies of the memory-enhancing effects of posttraining intra-BLA infusions of norepinephrine have generally used highly arousing training tasks such as inhibitory avoidance and contextual fear conditioning that use footshock (Berlau \& McGaugh, 2005; Ferry et al., 1999; Huff et al., 2005; Introini-Collison et al., 1991; LaLumiere et al., 2003) or water-maze training (Hatfield \& McGaugh, 1999). Such findings suggested that some consequences of high arousal might be essential in enabling noradrenergic activation of the BLA to modulate memory consolidation. The present findings clearly indicate that high levels of traininginduced arousal are not required for such modulating influences. Although the low degree of arousal produced by $3 \mathrm{~min}$ of object recognition training was insufficient to induce 24-h memory in saline-treated controls, such training conditions were sufficient to enable posttraining intra-BLA infusions of norepinephrine to induce $24-\mathrm{h}$ memory.

The finding that posttraining intra-BLA infusions of propranolol impaired object recognition memory induced by $10 \mathrm{~min}$ of training is consistent with extensive prior evidence that posttraining blockade of $\beta$-adrenoceptors impairs memory of many kinds of training experiences. A recent study reported that propranolol administered systemically blocks the enhancing effects of epinephrine on long-term object recognition memory (Dornelles, de Lima, Grazziotin, Presti-Torres, Garcia, Scalco, Roesler, \& Schröder, 2007).We previously found that intra-BLA infusions of propranolol administered after a novel taste (saccharine) attenuated latent inhibition of that taste when used to induce conditioned taste aversion $24 \mathrm{~h}$ later. However, such findings indicate that, unlike 3 min of object recognition training used in the present study, the novel taste training induced a 24-h taste memory. In comparison with the kinds of emotionally arousing training used in previous studies, the training conditions used in the present experiments induced relatively low levels of arousal (Okuda et al., 2004;

Roozendaal et al., 2006). Thus, the findings provide additional evidence that high levels of training-induced arousal are not required in order to observe BLA noradrenergic modulation of memory. We have previously found that the mild arousal induced by object recognition training is reduced by prior habituation to the training apparatus (Okuda et al., 2004; Roozendaal et al., 2006). Further, in rats given 3 min of training, posttraining systemic administration of the adrenocortical stress hormone corticosterone enhanced 24-h memory of non-habituated rats but failed to enhance memory of habituated rats, suggesting that traininginduced arousal is required to enable corticosterone enhancement of memory. The additional finding that intra-BLA infusions of propranolol blocked the memory enhancement seen in nonhabituated rats strongly suggested that corticosterone enhancement of memory requires arousal-induced noradrenergic activation of the BLA.

Thus, as the present experiments used non-habituated rats it seems likely that the object recognition training induced some degree of noradrenergic activation of the BLA. However, the $3 \mathrm{~min}$ of training was insufficient to induce $24 \mathrm{~h}$ memory. The finding that posttraining intra-BLA infusions of propranolol impaired 24-h memory of rats given 10 min of training provides additional evidence suggesting that noradrenergic activation of the BLA is essential in enabling long-term memory. Several prior studies have reported evidence that norepinephrine release within the amygdala depends on the degree of arousal. Footshock stimulation induces norepinephrine release in the amygdala and the amount varies directly with footshock intensity (Quirarte et al., 1998). Further, 24-h retention of inhibitory avoidance correlates highly with amount of norepinephrine released following training (McIntyre et al., 2002). As placement of rats into an unfamiliar inhibitory avoidance apparatus without footshock does not induce the release of a measurable amount of norepinephrine (McIntyre et 
al., 2002), it seems likely that norepinephrine levels induced by either 3 or $10 \mathrm{~min}$ of object recognition training are lower than those induced by more highly arousing experiences used in previous studies.

Although there is now extensive evidence that emotional arousal induces noradrenergic modulation of memory consolidation, the present findings suggest that such modulation is not likely to be a specific process activated only by highly arousing experiences that serve to ensure that we retain memories of those experiences. Rather, our findings suggest that norepinephrine release in the BLA provides an ongoing modulation of storage of recent experiences that vary in degree of emotional arousal induced. Intense arousal induces highly enduring memories (McGaugh, 2003) and, if such memories are of unpleasant experiences may contribute to the development of posttraumatic stress disorder (McGaugh, 2003; Pitman, Sanders, Zusman, Healy, Cheema, Lasko, Cahill, \& Orr, 2002). However, even very mild arousal enhances memory. Anderson, Wais and Gabrieli (2006) reported that viewing of mildly arousing pictures enhanced memories of neutral pictures seen a few seconds earlier. Further, PET and eventrelated fMRI imaging of amygdala activity during encoding of mildly arousing stimuli predict the subsequent memory of the arousing stimuli (Cahill, Haier, Fallon, Alkire, Tang, Keator, Wu, \& McGaugh, 1996; Canli, Zhao, Brewer, Gabrieli, \& Cahill, 2000;) and, importantly, propranolol blocks the enhanced retention otherwise induced by the arousing stimuli (Strange \& Dolan, 2004; van Stegeren, Goekoop, Everaerd, Scheltens, Barkhof, Kuijer, \& Rombouts, 2005).

In summary, the present findings clearly indicate that high levels of emotional arousal are not essential for modulation of memory consolidation induced by posttraining noradrenergic activation of the BLA. Our evidence indicates that posttraining intra-BLA infusions of norepinephrine enhance memory assessed at $24 \mathrm{~h}$ in animals trained with low-arousing conditions that otherwise do not induce long-term memory. These findings, considered together with prior evidence that more arousing training induces the release of norepinephrine within the amygdala, provide further evidence that noradrenergic activation of the BLA plays a central role in ensuring that emotionally significant experiences create lasting memories.

\section{Acknowledgements}

Supported by NIH Grant MH12526 (JLM) and NSF Grant IOB-618211 (BR)

\section{References}

Anderson AK, Wais PE, Gabrieli JD. Emotion enhances remembrance of neutral events past. Proceedings of the National Academy of Sciences of the United States of America 2006;103:1599-1604. [PubMed: 16434476]

Berlau DJ, McGaugh JL. Enhancement of extinction memory consolidation: The role of the noradrenergic and GABAergic systems within the basolateral amygdala. Neurobiology of Learning and Memory 2006;86:123-132. [PubMed: 16458544]

Bermudez-Rattoni F, Okuda S, Roozendaal B, McGaugh JL. Insular cortex is involved in consolidation of object recognition memory. Learning and Memory 2005;12:447-449. [PubMed: 16166398]

Cahill L, Haier RJ, Fallon J, Alkire M, Tang C, Keator D, Wu J, McGaugh JL. Amygdala activity at encoding correlated with long-term, free recall of emotional information. Proceedings of the National Academy of Sciences of the United States of America 1996;93:8016-8021. [PubMed: 8755595]

Canli T, Zhao Z, Brewer J, Gabrieli JD, Cahill L. Event-related activation in the human amygdala associates with later memory for individual emotional experience. Journal of Neuroscience 2000;20:RC99. [PubMed: 11000199]

Dornelles A, de Lima MNM, Grazziotin M, Presti-Torres J, Garcia VA, Scalco FS, Roesler R, Schröder N. Adrenergic enhancement of consolidation of object recognition memory. Neurobiology of Learning and Memory 2007;88:137-142. [PubMed: 17368053] 
Ferry B, Roozendaal B, McGaugh JL. Basolateral amygdala noradrenergic influences on memory storage are mediated by an interaction between beta- and alpha ${ }_{1}$-receptors. Journal of Neuroscience 1999;19:5119-5123. [PubMed: 10366644]

Hatfield T, McGaugh JL. Norepinephrine infused into the basolateral amygdale posttraining enhances retention in a spatial water maze task. Neurobiology of Learning and Memory 1999;71:232-239. [PubMed: 10082642]

Hatfield T, Spanis C, McGaugh JL. Response of amygdalar norepinephrine to footshock and GABAergic drugs using in vivo microdialysis and HPLC. Brain Research 1999;835:340-345. [PubMed: 10415392]

Huff NC, Wright-Hardesty KJ, Higgins EA, Matus-Amat P, Rudy JW. Context pre-exposure obscures amygdala modulation of contextual-fear conditioning. Learning and Memory 2005;12:456-460. [PubMed: 16204200]

Introini-Collison IB, Miyazaki B, McGaugh JL. Involvement of the amygdala in the memory-enhancing effects of clenbuterol. Psychopharmacology 1991;104:541-544. [PubMed: 1780426]

LaLumiere RT, Buen T-V, McGaugh JL. Posttraining intra-basolateral amygdale infusions of norepinephrine enhance consolidation of memory for contextual fear conditioning. Journal of Neuroscience 2003;23:6754-6758. [PubMed: 12890768]

McGaugh JL. Memory: A Century of Consolidation. Science 2000;287:248-251. [PubMed: 10634773]

McGaugh JL. Memory consolidation and the amygdala: A systems perspective. Trends in Neurosciences 2002;25:456-461. [PubMed: 12183206]

McGaugh, JL. Memory and Emotion: The Making of Lasting Memories. London: Weidenfeld and Nicolson The Orion House Group Ltd.; 2003. and New York: Columbia University Press.

McGaugh JL. The amygdala modulates the consolidation of memories of emotionally arousing experiences. Annual Review of Neuroscience 2004;27:1-28.

McGaugh JL, Roozendaal B. Role of adrenal stress hormones in forming lasting memories in the brain. Current Opinion in Neurobiology 2002;12:205-210. [PubMed: 12015238]

McIntyre CK, Hatfield T, McGaugh JL. Amygdala norepinephrine levels after training predict inhibitory avoidance retention performance in rats. European Journal of Neuroscience 2002;16:1223-1226. [PubMed: 12405982]

Miranda MI, LaLumiere RT, Buen TV, Bermudez-Rattoni F, McGaugh JL. Blockade of noradrenergic receptors in the basolateral amygdala impairs taste memory. European Journal of Neuroscience 2003;18:2605-2610. [PubMed: 14622162]

Okuda S, Roozendaal B, McGaugh JL. Glucocorticoid effects on object recognition memory require training-associated emotional arousal. Proceedings of the National Academy of Sciences of the United States of America 2004;101:853-858. [PubMed: 14711996]

Paxinos, G.; Watson, C. The Rat Brain in Stereotaxic Coordinates. 5th edn. San Diego: Academic Press; 2005.

Pitman RK, Sanders KM, Zusman RM, Healy AR, Cheema F, Lasko NB, Cahill L, Orr SP. Pilot study of secondary prevention of post-traumatic stress disorder with propranolol. Biological Psychiatry 2000;51:189-182. [PubMed: 11822998]

Quirarte GL, Galvez R, Roozendaal B, McGaugh JL. Norepinephrine release in the amygdala in response to footshock and opioid peptidergic drugs. Brain Research 1998;808:134-140. [PubMed: 9767150]

Roozendaal B, McGaugh JL. Amygdaloid nuclei lesions differentially affect glucocorticoid-induced memory enhancement in an inhibitory avoidance task. Neurobiology of Learning and Memory 1996;65:1-8. [PubMed: 8673403]

Roozendaal B, McGaugh JL. Basolateral amygdala lesions block the memory-enhancing effect of glucocorticoid administration in the dorsal hippocampus of rats. European Journal of Neuroscience 1997;9:76-83. [PubMed: 9042571]

Roozendaal B, Okuda S, Van der Zee EA, McGaugh JL. Glucocorticoid enhancement of memory requires arousal-induced noradrenergic activation in the basolateral amygdala. Proceedings of the National Academy of Sciences of the United States of America 2006;103:6741-6746. [PubMed: 16611726]

Strange BA, Dolan RJ. $\beta$-adrenergic modulation of emotional memory-evoked human amygdala and hippocampal responses. Proceedings of the National Academy of Sciences of the United States of America 2004;101:11454-11458. [PubMed: 15269349] 
van Stegeren AH, Goekoop R, Everaerd W, Scheltens P, Barkhof F, Kuijer JP, Rombouts SA. Noradrenaline mediates amygdala activation in men and women during encoding of emotional material. Neuroimage 2005;24:898-909. [PubMed: 15652324] 

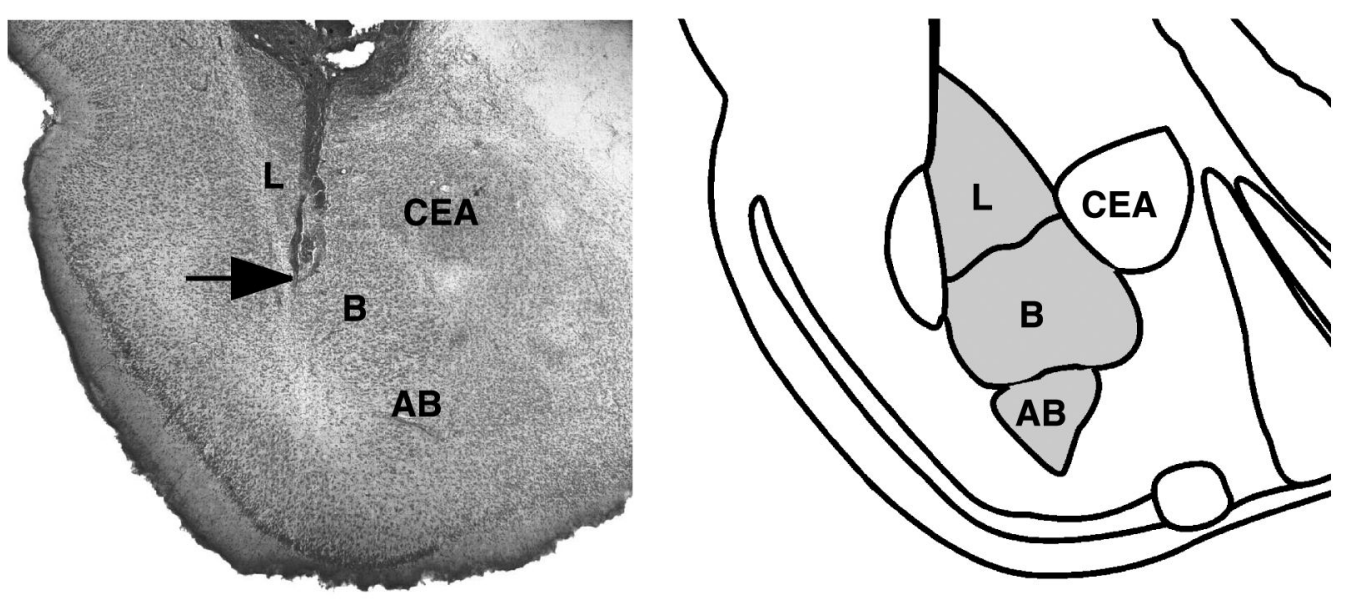

Figure 1.

Representative photomicrograph illustrating placement of a cannula and needle tip in the BLA. Arrow points to needle tip. The gray area in the diagram represents the different nuclei of the BLA: the lateral nucleus (L), basal nucleus (B) and accessory basal nucleus (AB). CEA, central nucleus of the amygdala. 

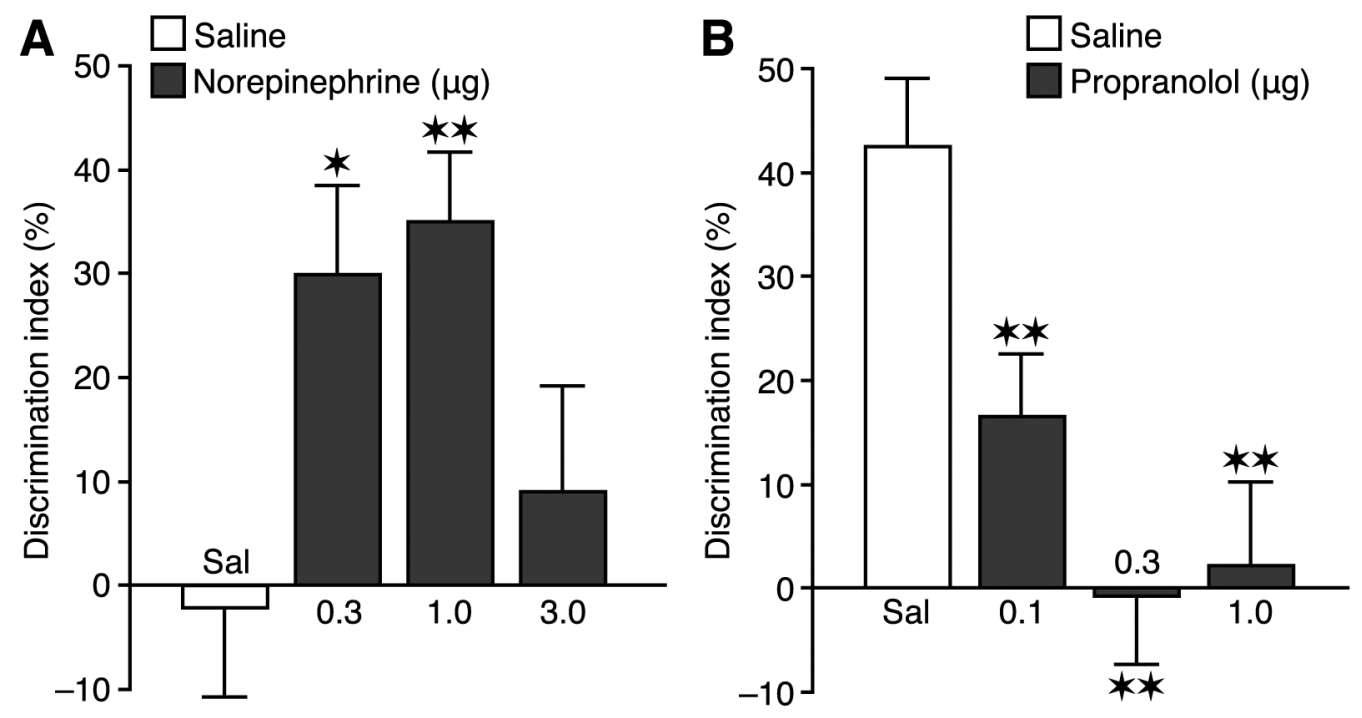

Figure 2.

Noradrenergic activation of the BLA modulates consolidation of object recognition memory. (A) Enhancing effects of posttraining intra-BLA infusions of norepinephrine on 24-h object recognition memory. Saline-infused controls displayed no evidence of memory of 3 min of training. The retention performance of groups given 0.3 or $1.0 \mu \mathrm{g}$ of norepinephrine was significantly better than that of the saline controls. Data are presented as discrimination index (mean \pm SEM; see main text). (B) Impairing effects of posttraining intra-BLA infusions of propranolol on 24-h object recognition memory. All groups received 10 min of training. Salineinfused controls displayed significant memory and propranolol produced dose-dependent impairment of memory. The performance of all three propranolol groups differed significantly from that of the saline controls. $* P<0.05$; ** $P<0.01$. $\mathrm{N}=7-9$ rats per group. 